\title{
Données préliminaires sur la diversité du zooplancton du lac Nokoué (Sud-Bénin)
}

\author{
Delphine ADANDEDJAN, Expédit MAKPONSE, Lambert C. HINVI, Philippe LALEYE \\ Laboratoire d'Hydrobiologie et d'Aquaculture (LHA)/Faculté des Sciences Agronomiques (FSA)/Université d'Abomey- \\ Calavi (UAC) 01 BP 521 Cotonou Bénin. Tél : 97339765 ; Email : adandedjandelph@gmail.com .
}

Original submitted in on 21st April 2017. Published online at www.m.elewa.org on 31st July 2017 https://dx.doi.org/10.4314/jab/v115i1.7

\begin{abstract}
RESUME
Objectif : L'étude de diversité et la répartition des organismes zooplanctoniques du Lac Nokoué a été menée. Méthodologie et résultats : Pour la compréhension de cette composante importante de l'écosystème, un échantillonnage mensuel a été effectué sur 4 mois (juin à septembre 2015). Au cours de l'étude, 40 échantillons de zooplancton collectés au moyen d'un filet à plancton, dans 10 stations sont analysés. Au total, 31 taxa constitués de Copépodes, de Rotifères et de Cladocères ont été identifiés puis dénombrés. Les rotifères ont été le groupe le plus diversifié, avec une forte participation de la famille des Brachionidae. Sur le plan quantitatif, les nauplii de Copépodes ont été dominants à toutes les stations et en toutes périodes de l'étude. En général, une faible densité de zooplancton a été obtenue dans le lac. Toutefois, le mois de juillet et la station de Ganvié ont présenté des richesses taxinomiques et des densités élevées. Cependant les indices biologiques calculés ont indiqué une mauvaise répartition des individus dans la diversité zooplanctonique disponible. De plus, l'analyse de redondance réalisée entre les variables physico-chimiques et les principaux taxa a fait ressortir le taux de nitrate et celui d'ammonium comme les principaux paramètres qui déterminent les Copépodes et les Rotifères.

Conclusion et application des résultats : Ces données bien que préliminaires ont montré que la distribution dans le lac du zooplancton est régie par la présence de matières organiques. Toutefois, une étude conduite sur une période plus ou moins longue combinant les facteurs physico-chimiques et la collecte du zooplancton contribuera davantage à la compréhension de la dynamique de cette communauté biologique du lac

Mots clés : Zooplancton, diversité, densité, distribution, Canoco, lac Nokoué.
\end{abstract}

\begin{abstract}
Objective: The study of the diversity and the distribution of zooplanktonic organisms of Nokoue Lake was conducted.

Methodology and Results: For an understanding of this important component of the ecosystem, monthly sampling was carried out for four months June to September 2015). During the study, 40 zooplankton samples collected by plankton net, in 10 stations are analyzed. A total of 31 taxa consisting of Copepods, Rotifers and Cladocerans were identified and counted. Rotifers are the most diverse group, with strong participation of Brachionidae family. Quantitatively, Copepod nauplii were dominant at all stations and in all periods of the study. In general, a low density of zooplankton was obtained in the lake. However, July month and Ganvié station have registered high taxonomic richness and densities than other months and stations sampled.
\end{abstract}



(Sud-Bénin)

However, the calculated biological indices indicate a distribution of individuals in the zooplankton diversity. In addition, the redundancy analysis carried out between the physico-chemical variables and key taxa highlighted the nitrate levels and ammonium levels, such as parameters that determine Copepods and Rotifers in this ecosystem. These data, although preliminary, showed that the distribution of the organisms is controlled by the organic matters.

Conclusion and application of results: However, studies that combine long duration, physico chemical factors and the zooplancton would more contribute to the understanding of the dynamic of these organisms.

Keywords: zooplankton, species diversity, density, distribution, Canoco, Lake Nokoué.

\section{INTRODUCTION}

Les écosystèmes aquatiques abritent un ensemble diversifié d'organismes en interaction entre eux et avec leur milieu de vie. Chacun de ces organismes, à travers sa niche écologique et indépendamment de sa taille, joue un rôle spécifique dans le fonctionnement et l'équilibre de l'écosystème. Mais dans les pays en voie de développement, l'intégrité de la plupart des cours et plans d'eau est menacée par l'évacuation des déchets des villes et des industries. Ces actions anthropiques conduisent à coup sûr à une dégradation de la qualité de l'eau, une modification des peuplements et le plus souvent, une diminution de la biodiversité. L'exemple concret est aujourd'hui la diminution de la taille de maturité des poissons, la baisse des captures et même l'abandon du secteur de pêche par les riverains qui se convertissent en conducteurs de moto taxi « Zémidjan » au Bénin. (Adandédjan, 2012) Alors, dans une perspective de gestion durable de la ressource, il est impérieux d'étudier ces écosystèmes, dans toutes leurs composantes, et de maîtriser leur fonctionnement. De nombreuses études ont été déjà amorcées au Bénin, et ont tenté de mettre en exergue l'état écologique des écosystèmes aquatiques en se basant sur le compartiment benthique (Gnohossou, 2006 ; Adandédjan, 2012). Cependant, l'étude des organismes aquatiques a souvent occulté les microorganismes qui constituent la production primaire et même secondaire des écosystèmes. II s'agit du compartiment planctonique composé d'organismes très variés, aussi bien par leur taille que par leur morphologie, mais également par leurs rôles dans les écosystèmes aquatiques dont fait partie le zooplancton. Le rôle écologique de ces organismes est fondamental en ce sens qu'il constitue les premiers chaînons trophiques avec le phytoplancton producteur de dioxygène et le zooplancton base de la production primaire. En effet, Haberman (1998) a estimé à plus de $60 \%$ la production primaire lacustre transférée par le zooplancton aux alevins de poissons. Principale source de protéine pour les alevins et les juvéniles de poissons, le zooplancton par son mode de vie, son environnement et ses réactions aux perturbations et aux modifications de l'eau, est devenu un excellent indicateur du changement climatique (Brassard, 2009). Établir un lien de causalité entre les perturbations dans l'environnement et les divers paramètres biotiques est fondamental afin de comprendre l'impact réel des activités anthropiques sur les communautés écologiques des cours et plans d'eau (Adandédjan, 2012). Ainsi, le zooplancton, abondant dans les eaux douces et très sensibles aux variations des conditions de son milieu, pourrait être utilisé pour établir un tel lien et servir par conséquent d'outils d'évaluation de l'état de santé des écosystèmes, donc de bioindicateurs (Zébazé Togouet, 2000). Pour Arfi et Patriti (1987), une approche efficace des quantités d'énergie et de matière fournies par le zooplancton à un système, peut être développée à travers l'inventaire des espèces présentes et l'étude de leur dynamique dans l'espace et dans le temps. Au Bénin, les recherches sur les communautés zooplanctoniques restent fragmentaires. Dans le lac Nokoué, Gnohossou (2002) a identifié quelques groupes de zooplancton parmi lesquels les ostracodes, les copépodes, les cladocères, les rotifères, les nauplii et les protozoaires, notamment les Tintinnidae. Une étude ponctuelle mais plus large a été effectuée par Abou (2001) sur la connaissance de la richesse spécifique du zooplancton des zones humides du Sud-Bénin. II a dénombré 20 espèces de rotifères et 8 espèces de cladocères. II n'existe 

(Sud-Bénin)

quasiment pas au Bénin de travaux spécifiquement basés sur l'étude approfondie du zooplancton en général, et celui de lac Nokoué en particulier. La présente étude dont le thème s'intitule « Diversité et distribution des communautés du zooplancton dans le lac Nokoué au Sud-Bénin» s'inscrit dans la dynamique de fournir une connaissance de base sur l'évolution spatio-temporelle des communautés de

\section{MATÉRIEL ET MÉTHODES}

Milieu d'étude : Le lac Nokoué, le plus grand lac du Bénin, couvre une superficie de $150 \mathrm{~km}^{2}$ à l'étiage. Situé au sud-est du pays, il est compris entre les parallèles $6^{\circ} 20^{\prime}$ et $6^{\circ} 30^{\prime}$ Nord et les méridiens $2^{\circ} 20^{\prime}$ et $2^{\circ} 35^{\prime}$ Est. S'étendant sur les départements de l'Ouémé, de l'Atlantique et du Littoral, il est limité à l'Ouest par le plateau d'Abomey-Calavi, à l'Est par la lagune de PortoNovo, au Nord par la plaine d'inondation du fleuve Ouémé et de la rivière Sô puis au Sud par la ville de Cotonou, comme l'indique la Figure 1. Le chenal de Cotonou et le canal de Totchè relient respectivement le lac à l'océan Atlantique et à la lagune de Porto-Novo. Le lac Nokoué a une longueur moyenne de $20 \mathrm{~km}$ dans sa direction est-ouest et une largeur de $11 \mathrm{~km}$ dans sa direction nord-sud. Le régime hydrologique du lac Nokoué est caractérisé par une faible crue de mai à juin qui correspond à la grande saison des pluies au sud du Bénin, une forte crue de septembre à novembre correspondant à la petite saison des pluies et aux apports d'eau par le fleuve Ouémé et la rivière Sô, et une période d'étiage de décembre à mars et enfin une petite saison sèche de mi-juillet à mi-septembre. La durée des crues varie suivant que le chenal de Cotonou est fermé ou ouvert et selon l'importance des pluies. En effet, le chenal de Cotonou tend à se fermer par l'accumulation du sable d'origine marine, mais il s'ouvre périodiquement sous l'effet des tempêtes. Cette alternance d'isolement et de communication directe avec la mer, jointe à l'effet des zooplancton de cet écosystème et son niveau de pollution. L'objectif général de notre étude est de caractériser la communauté zooplanctonique du lac Nokoué en relation avec la qualité de l'eau du lac Nokoué. Plus spécifiquement il s'agit (i) d'inventorier la faune zooplanctonique du lac; (ii) d'étudier la distribution des communautés de zooplancton en relation avec les facteurs environnementaux.

crues naturelles du fleuve Ouémé et de la rivière Sô, provoque des variations très importantes de salinité dans le lac. Actuellement, environ 1500000 personnes, y compris les habitants de la ville de Cotonou, dépendent du lac (Gnohossou, 2006). Ceux qui vivent sur le lac ont édifié des habitations sur pilotis et sont essentiellement pêcheurs. Ce lac est riche en diversité ichtyologique avec 51 espèces de poisson (Lalèyè et al. (2003). Cette richesse en poisson fait du lac un pôle d'attraction tant pour les pêcheurs autochtones qu'allochtones et utilisant d'engins et techniques de pêche variés. L'excessivité des activités anthropiques exercée par les populations sur le lac a induit des pollutions non des moindres dans le lac. Malgré l'intervention de l'état, tous les efforts de gestion durable promulgués sont voués à l'échec. Les techniques et pratiques onéreuses de pêche comme le système " acadja » et le filet «Dokpokonou » continuent d'être utilisées sur ce plan d'eau. Dans le cadre de cette étude dix stations ont été choisies (Figure 1). Elles ont été choisies en considérant l'aire du lac, l'importance des activités anthropiques et l'accès facile. Soulignons que huit de ces stations retenues ont déjà servi de base pour l'analyse de données écologiques dans le domaine ichtyologique et de macroinvertébrés. Quatre (04) campagnes de prélèvement ont été effectuées suivant une périodicité mensuelle sur deux saisons : petite saison sèche (juin, juillet) et petite saison pluvieuse (août, septembre) en 2015. 


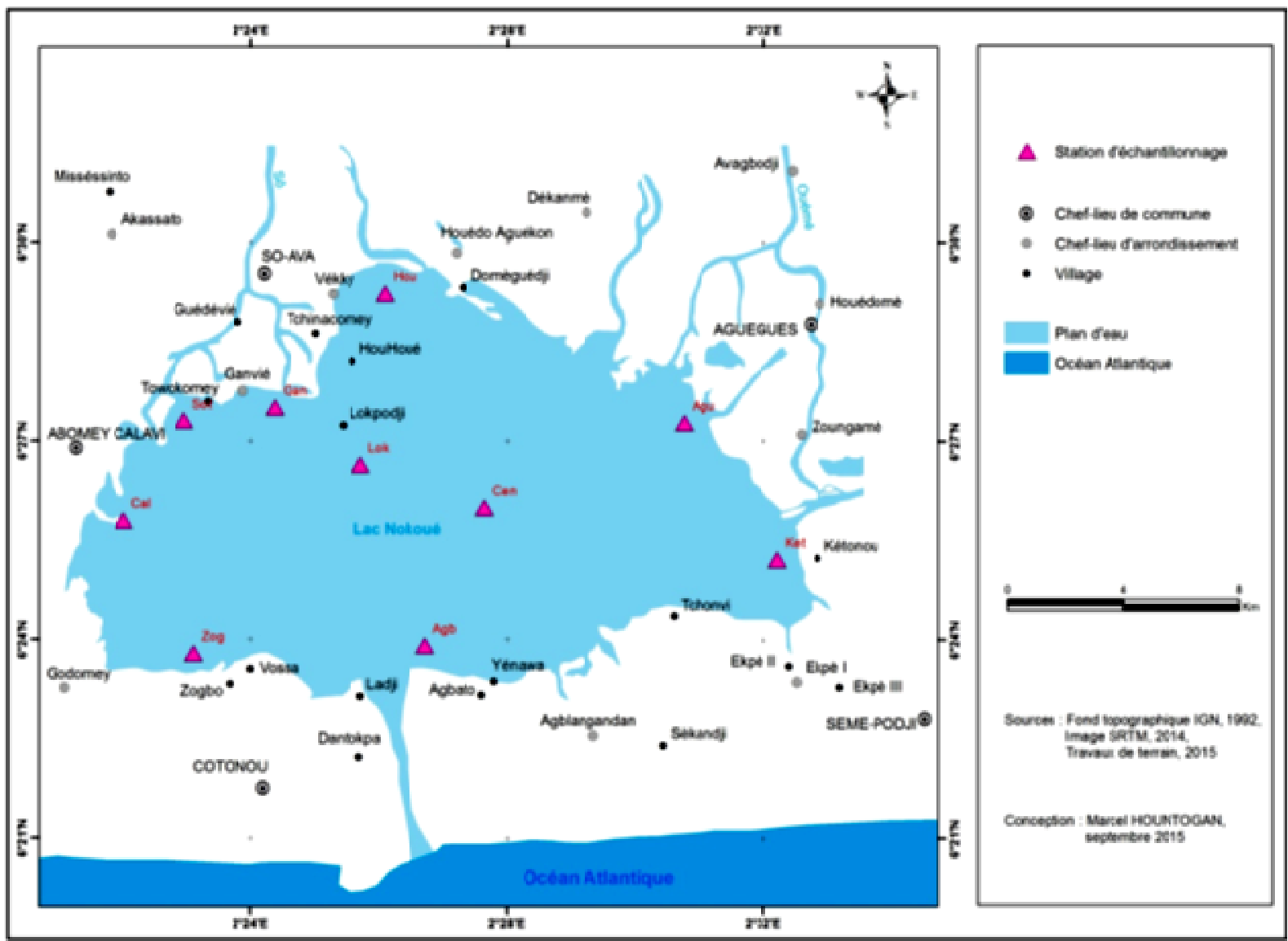

Figure 1 : Carte du Lac Nokoué et stations d'échantillonnage.

\section{Techniques de prélèvement et d'analyse des échantillons}

- $\quad$ Paramètres physico-chimiques : Huit paramètres physico-chimiques à savoir la température de l'eau, la transparence, la profondeur, le $\mathrm{pH}$, l'oxygène dissout, le taux de saturation en oxygène, la salinité et la conductivité ont été mesurés in situ entre $7 \mathrm{~h}$ et $10 \mathrm{~h}$. La profondeur et la transparence ont été mesurées à l'aide du disque de Secchi muni d'une corde graduée. La température, le $\mathrm{pH}$, l'oxygène dissous, le pourcentage de saturation en oxygène, la salinité et la conductivité électrique ont été mesurés à l'aide d'un conductimètre multifonction. Des échantillons d'eau destinés à l'analyse des sels dissouts nitrites, nitrates et phosphates, ont été ramenés au Laboratoire de Contrôle de Qualité des Eaux et Aliments (LCQEA) de la Direction Nationale de la Santé Publique (DNSP où les concentrations ont été déterminées au spectrophotomètre. Notons que ces prélèvements d'eau ont été faits à contre-courant, en prenant soin de ne pas faire de bulles, dans des flacons à double bouchage en polyéthylène de $1500 \mathrm{~mL}$, puis transportés au laboratoire dans une enceinte réfrigérée.
- Prélèvement du zooplancton : Un filet à plancton (diamètre d'ouverture : $30 \mathrm{~cm}$; maille : $30 \mu \mathrm{m}$ ) a été utilisé pour le prélèvement du zooplancton. Avant de procéder à chaque échantillonnage, le filet a été rincé avec l'eau du lac afin d'en déloger toute matière qui pourrait s'y trouver. Aussi, à la fin de chaque échantillonnage, le filet et le godet ont été rincés avec l'eau du lac avant de passer à un autre site d'échantillonnage. A chaque station d'échantillonnage et à une profondeur inférieure à $50 \mathrm{~cm}$, le filet à plancton muni d'entonnoir, a été trainé horizontalement dans l'eau sur une distance d'au moins $10 \mathrm{~m}$. Le processus est répété 5 fois à chaque station, jusqu'à filtration d'un volume total de $100 \mathrm{~L}$ d'eau. Ensuite le filet est rincé à l'eau de l'échantillon dans le sens contraire de celui de la filtration pour ne perdre aucun organisme ; ce qui permet d'avoir un échantillon de $150 \mathrm{~mL}$ après filtration concentré dans un bocal de $200 \mathrm{~mL}$. Pour l'étude quantitative, le filet a été trainé sur une distance de $15 \mathrm{~m}$, soit un volume de $740 \mathrm{~L}$ d'eau filtrée. Les échantillons concentrés, ont été ensuite fixés avec du formol à $5 \%$. 

(Sud-Bénin)

- Identification et dénombrement du zooplancton : L'identification et le dénombrement ont été réalisés au Laboratoire d'Hydrobiologie et d'Aquaculture (LHA) de la FSA, au moyen d'un microscope optique marque Olympus CK2 UL WCD 0.30 à l'aide de clés et ouvrages d'identification de Durand et Lévêque (1980), Pourriot (1980); Rey et Saint-Jean (1980); Dussart (1980); Pourriot et Francez (1986), Segers (1996), Zébazé Togouet (2000) et Fernando (2002) suivant les techniques classiques. L'étude quantitative $\mathrm{du}$ zooplancton a été faite sur un prélèvement de $1 \mathrm{~mL}$ d'échantillon bien homogénéisé, avec trois (03) répétitions (soit au total $3 \mathrm{~mL}$ de chaque échantillon). Compte tenu des clés et ouvrages disponibles, l'identification et le comptage des rotifères, des cladocères, des copépodes et des nauplii ont été effectués. La densité des individus a été calculée avec la formule suivante utilisée par Agadjihouèdé et al. (2010) : $\mathrm{D}=(\mathrm{n} / \mathrm{v} 1) \times(\mathrm{v} 2 / \mathrm{v} 3)$ avec $\mathrm{n}=$ nombre d'individus comptés, $\mathrm{v}_{1}=$ volume du filtrat prélevé $(3 \mathrm{~mL}), \mathrm{v}_{2}=$ volume du filtrat concentré (volume échantillon), $\mathrm{v}_{3}=$ volume d'eau filtrée (740 L).

Analyse et traitement des données : Les valeurs des paramètres mesurés sont présentées dans un tableau de synthèse. Les données biologiques obtenues ont servi à déterminer la richesse taxinomique et calculer différents indices de diversité permettant de caractériser la composition et l'évolution du zooplancton. La densité des organismes (D) ou le nombre d'individus des organismes par unité de volume a été calculé. La fréquence d'occurrence $(\%$ Occ) des espèces a été aussi déterminée par la relation : \%Occ $=(\mathrm{Pa} / \mathrm{P}) \times 100$ avec $\mathrm{Pa}=$ nombre total de prélèvements contenant l'espèce prise en considération; $P$, le nombre total de prélèvements faits. La clé de Dajoz (1982), a été utilisée pour classer les espèces : les espèces constantes $(\%$ Occ $\geq 50 \%$ ), les espèces accessoires $(25 \%<\%$ Occ $<50 \%)$ et les espèces accidentelles ( $\%$ Occ $\leq 25 \%)$. Parmi les espèces accidentelles, celles qui ont moins de $5 \%$ d'occurrence sont des espèces rares. Les indices de diversité alpha ont été calculés pour affiner la structuration du peuplement :

- Indice de Shannon-Wiener ( $H^{\prime}$ ) :L'indice de diversité de Shannon-Wiener a rendu compte de la diversité des espèces qui composent les peuplements dans le milieu. Sa formule est: $H^{\prime}=-\Sigma\left[(n i / N) \times \log _{2}(n i / N)\right]$. H' représente la diversité spécifique en bits, ni l'effectif de l'espèce i, N le nombre total d'individus en considérant toutes les espèces et $\log _{2}$ le logarithme en base 2 .

- Indice d'équitabilité de Piélou (Eq) : L'indice Eq de Piélou a permis de mesurer l'équitabilité (ou équirépartition) des espèces du peuplement par rapport à une répartition théorique égale pour l'ensemble des espèces. II s'obtient par la formule : $\mathrm{Eq}=\mathrm{H}^{\prime} / \log _{2} \mathrm{~S}$

$\mathrm{H}^{\prime}$ est l'indice de Shannon-Wiener, $\log _{2}$, le logarithme en base 2 et $S$ le nombre d'espèces présentes. L'indice Eq varie de 0 (dominance d'une seule espèce) à 1 (équirépartition des individus dans les peuplements).

- Indice de Simpson (C) :L'indice d'abondance de Simpson a permis d'évaluer le déséquilibre, en termes d'individu, au sein du peuplement. II se calcule par la formule : $\mathrm{C}=\Sigma(\mathrm{ni} / \mathrm{N}){ }^{2}$ ni l'effectif de l'espèce i, $\mathrm{N}$ le nombre total d'individus en considérant toutes les espèces. Plus sa valeur se rapproche de $100 \%$, plus il y a dominance des individus de quelques taxa.

Traitement statistique des données: Analyse de Redondances (RDA): L'Analyse Canonique des Redondances a été effectuée pour identifier les paramètres du milieu qui expliquent une partie de la variance dans la structure des communautés du zooplancton. Cette méthode réalise les analyses de gradients indirectes habituelles comme l'analyse en composantes principales (Adandédjan, 2012). Ce module permet d'étudier la relation entre deux ensembles de variables. Elle a permis ainsi de mettre en relation les paramètres physico-chimiques, la présence et l'abondance des principales espèces $(\mathrm{Dr}>5 \%) \mathrm{du}$ zooplancton dans le lac. Cette analyse a été réalisée avec le logiciel CANOCO 4.5 (CANOnical Community Ordination version 4.5). L'analyse en RDA (ReDundancy Analysis) s'est révélé la plus appropriée. La pertinence de l'analyse est d'abord vérifiée grâce à un test de permutation de Monte-Carlo réalisé sur 499 permutations aléatoires.

Tests de comparaison : Le test statistique d'analyse de variance a été utilisé pour évaluer les différences entre stations et mois d'échantillonnage, la richesse et l'abondance absolue des taxa. Le test non paramétrique de Kruskal-Wallis a été utilisé. Les différents tests statistiques ont été exécutés sous le logiciel STATISTICA version 4.5 . 

(Sud-Bénin)

RÉSULTATS

Variables environnementales : Les valeurs moyennes et extrêmes des paramètres environnementaux du lac sont présentées dans le tableau 1.

Tableau1 : Valeurs moyennes et extrêmes des paramètres physico-chimiques de l'eau du lac (période de juin à septembre 2015).

\begin{tabular}{|c|c|c|c|c|c|c|c|c|c|c|c|c|c|}
\hline & Temp & Trans & Prof & pH & Sali & Cond & TDS & O2 di & Sat02 & P043- & NH4+ & NO3- & NO2- \\
\hline Min & 25,1 & 12 & 0,22 & 7,3 & 0,13 & 1,5 & 0,08 & 2,31 & 30 & 0,01 & 0,1 & 0,009 & 0,001 \\
\hline Max & 28,2 & 87 & 2,3 & 8,9 & 15,5 & 26,4 & 25,5 & 5,02 & 60 & 1,15 & 0,9 & 0,5 & 0,092 \\
\hline Moy & 26,54 & 43,42 & 1,04 & 8,03 & 6,92 & 12,91 & 12,77 & 3,40 & 42,69 & 0,21 & 0,32 & 0,14 & 0,03 \\
\hline
\end{tabular}

Légende: Min = minimum; Max = maximum; Moy = Moyenne; Trans = transparence $(\mathrm{cm}) ;$ Cond= conductivité $(\mathrm{Ms} / \mathrm{cm})$ Sali = salinité $(\mathrm{mg} / \mathrm{L})$, Temp $=$ température $\left({ }^{\circ} \mathrm{C}\right)$, Prof $=$ profondeur $(\mathrm{m}), 02 \mathrm{di}=$ Oxygène dissous $(\mathrm{mg} / \mathrm{L})$, SatO2 $=$ taux de saturation en oxygène $(\%), \mathrm{NO}^{-}=$Nitrate $(\mathrm{mg} / \mathrm{L}), \mathrm{NO}^{-}=$nitrite $(\mathrm{mg} / \mathrm{L}), \mathrm{NH}^{+}=$ammonium $(\mathrm{mg} / \mathrm{L}), \mathrm{PO}^{3-}=$ orthophosphate $(\mathrm{mg} / \mathrm{L})$.

Composition faunistique et densités : Trente et un (31) taxa de zooplancton ont été récoltés au cours de la période de l'étude (Tableau 2). Parmi ces taxa, on distingue 4 taxa de copépodes, 3 taxa de cladocères et 24 taxa de rotifères. Les rotifères sont les plus diversifiés à tous les niveaux taxonomiques et ont renfermé 12 familles et 16 genres comme le montre la figure 2 suivante. Au total, 1988,9 individus/L ont été recueillies au cours de l'étude. La figure 2 donne les densités des taxa par groupe obtenu au cours de l'étude. Ce sont les nauplii de copépodes qui ont présenté les densités les plus élevées $(1527,7$ ind/L). Les copépodes et les rotifères avec respectivement 261,5 et 198,3 ind/L ont été aussi des groupes importants de l'écosystème. Deux taxa ont une densité relative $\mathrm{Dr} \geq 5 \%$; ce sont des taxa prépondérants du lac durant l'étude. Parmi ces taxa, on peut citer le taxon indéfini, nauplii de copépode qui ont représenté près des $77 \%$ des effectifs des organismes échantillonnés. Ensuite, ce sont les cyclopoïdes avec une densité relative de $8 \%$.
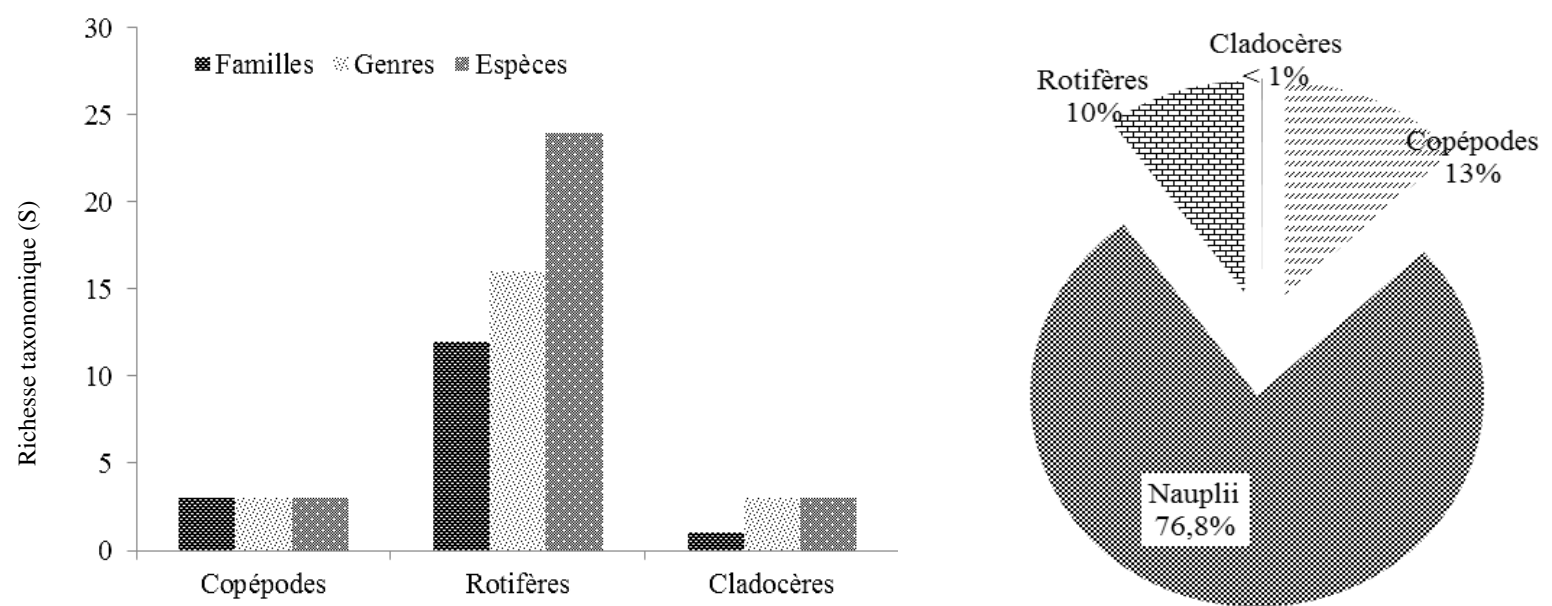

Figure 2 : Richesse taxinomique des organismes zooplanctoniques (à gauche) et Densités des organismes (à droite) durant l'étude. 

(Sud-Bénin)

Tableau 2 : Inventaire des organismes zooplanctoniques obtenus durant l'étude dans le lac.

\begin{tabular}{|c|c|c|c|c|c|c|c|c|c|c|c|c|c|}
\hline Taxa & Famille & Espèce & $\mathrm{Ca}$ & $\mathbf{G a}$ & So & Lo & Ho & $\mathrm{Ag}$ & $\mathrm{Ke}$ & $\mathrm{Ag}$ & $\mathrm{Ce}$ & Zo & $\% 0$ \\
\hline \multirow{4}{*}{$\begin{array}{l}\text { Copépo } \\
\text { des }\end{array}$} & Calanoida & Calanoïde spp. & $x$ & $\mathrm{x}$ & $\mathrm{x}$ & $x$ & $x$ & $\mathrm{x}$ & $\mathrm{x}$ & $\mathrm{x}$ & $\mathrm{x}$ & $x$ & 92 \\
\hline & Cyclopida & Cyclopoïde spp. & $\mathrm{x}$ & $x$ & $\mathrm{x}$ & $x$ & $x$ & $\mathrm{x}$ & $\mathrm{x}$ & $\mathrm{x}$ & $\mathrm{x}$ & $\mathrm{x}$ & 97, \\
\hline & Harpactic & Harpactioïde spp. & $\mathrm{x}$ & $\mathrm{x}$ & $\mathrm{x}$ & $\mathrm{x}$ & $\mathrm{x}$ & $\mathrm{x}$ & $\mathrm{x}$ & $\mathrm{x}$ & $\mathrm{x}$ & $\mathrm{x}$ & 82, \\
\hline & & Nauplius de copépode & $\mathrm{x}$ & $\mathrm{x}$ & $\mathrm{x}$ & $x$ & $x$ & $\mathrm{x}$ & $\mathrm{x}$ & $\mathrm{x}$ & $\mathrm{x}$ & $\mathrm{x}$ & 100 \\
\hline \multirow{24}{*}{$\begin{array}{l}\text { Rotifère } \\
\mathrm{s}\end{array}$} & Collotheci & Anuraeopsis navicula & & $x$ & - & - & - & - & - & - & - & - & 5 \\
\hline & Asplanch & Asplanchna girodi & & $\mathrm{x}$ & $\mathrm{x}$ & - & - & - & - & $\mathrm{x}$ & - & $\mathrm{x}$ & 22, \\
\hline & \multirow{10}{*}{$\begin{array}{l}\text { Brachioni } \\
\text { dae }\end{array}$} & Brachionus angularis & & $\mathrm{x}$ & $\mathrm{x}$ & $\mathrm{x}$ & - & - & - & - & - & - & 20 \\
\hline & & Brachionus calyciflorus & & $x$ & $\mathrm{x}$ & - & - & - & - & - & - & - & 12 \\
\hline & & Brachionus dimidiatus & $\mathrm{x}$ & $\mathrm{x}$ & $\mathrm{x}$ & $\mathrm{x}$ & - & - & $\mathrm{x}$ & - & - & - & 32 \\
\hline & & Brachionus falcatus & & $\mathrm{x}$ & $\mathrm{x}$ & $x$ & - & - & - & - & - & - & 20 \\
\hline & & Brachionus palulus & & - & - & - & - & - & $\mathrm{x}$ & - & - & - & 5 \\
\hline & & Brachionus plicatilis & $x$ & $x$ & $x$ & $\mathrm{x}$ & $\mathrm{x}$ & $x$ & $x$ & - & - & $x$ & 65 \\
\hline & & Epiphanes clavulata & & $x$ & $x$ & $x$ & - & - & $x$ & - & - & - & 25 \\
\hline & & Keratella cochlearis & & $x$ & $\mathrm{x}$ & $x$ & $x$ & - & - & $\mathrm{x}$ & - & - & 40 \\
\hline & & Keratella lenzi & & $x$ & $\mathrm{x}$ & - & - & - & - & - & - & - & 12, \\
\hline & & Keratella tropica & & $\mathrm{x}$ & $\mathrm{x}$ & $\mathrm{x}$ & - & - & $\mathrm{x}$ & - & - & - & 27, \\
\hline & Notomma & Cephalodella & & $\mathrm{x}$ & - & - & - & - & - & - & - & - & 7,5 \\
\hline & Conochili & Conochilus sp. & & $x$ & $x$ & $x$ & - & - & - & - & - & - & 17, \\
\hline & Euchlanid & Euchlanis & & $x$ & $x$ & - & - & $x$ & $\mathrm{x}$ & - & - & - & 22, \\
\hline & \multirow{4}{*}{$\begin{array}{l}\text { Testudine } \\
\text { llidae }\end{array}$} & Filinia Iongiseta & & $\mathrm{x}$ & - & - & - & - & $\mathrm{x}$ & - & - & - & 15 \\
\hline & & Filinia opoliensis & & $\mathrm{x}$ & - & - & - & - & - & - & - & - & 5 \\
\hline & & Hexarthra intermedia & & $x$ & - & - & - & - & - & - & - & - & 5 \\
\hline & & Testudinella & & - & - & $x$ & $x$ & - & $x$ & - & - & $x$ & 20 \\
\hline & Lecanida & Lecane leontina & & $\mathrm{x}$ & $\mathrm{x}$ & - & - & $\mathrm{x}$ & - & - & - & - & 20 \\
\hline & Colurellid & Lepadella patella & & $\mathrm{x}$ & $\mathrm{x}$ & - & - & $\mathrm{x}$ & - & - & - & - & 22, \\
\hline & Mytilinida & Mytilina mucronata & & $x$ & - & - & - & - & - & - & - & - & 5 \\
\hline & Philodinid & Philodina sp. & $x$ & $\mathrm{x}$ & $\mathrm{x}$ & $x$ & $x$ & $\mathrm{x}$ & $\mathrm{x}$ & $\mathrm{x}$ & $x$ & - & 62 \\
\hline & Synchaeti & Polyarthra & & $x$ & $\mathrm{x}$ & $x$ & $x$ & - & - & - & - & - & 20 \\
\hline \multirow{3}{*}{ Cladoc } & \multirow{3}{*}{$\begin{array}{l}\text { Daphniida } \\
\text { e }\end{array}$} & Ceriodaphnia & & - & $x$ & - & - & - & - & - & - & - & 5 \\
\hline & & Daphnia sp. & & $\mathrm{x}$ & - & - & - & - & - & - & - & - & 5 \\
\hline & & Scapholeberis kingi & $x$ & - & - & - & $x$ & - & - & - & - & - & 10 \\
\hline
\end{tabular}

Occurrence des taxa : Le pourcentage d'occurrence des taxa a été calculé pour déterminer les taxa selon leur constance dans l'écosystème (Tableau 2). Au total, 6 espèces ont été constantes soit $19,35 \%$ de la richesse totale. II s'agit des Copépodes, Calanoïde spp., Cyclopoïde spp., Harpactioïde spp., et les nauplii, des Rotifères Brachionus plicatilis et de Philodina sp.. Ensuite, 3 espèces constituant $9,67 \%$ de la composition taxinomique ont été accessoires. II s'agit de : Keratella tropica, Keratella cochlearis et Brachionus dimidiatus. Enfin, 70,96\% des taxa représentés par 22 espèces ont été accidentels au cours de l'étude. Parmi ces taxa, 7 espèces ont présenté des occurrences de $5 \%$.

Variations spatiales de la richesse et de la densité des organismes : La figure 3 a présenté les variations spatiales de la richesse taxinomique et de la densité du zooplancton du lac. Les stations de Ganvié, Sotchanhoué et Lokpodji avec des richesses taxonomiques comprises entre 15 et 27 , se distinguent des autres stations dont la richesse taxonomique varie, en général, de 5 à 10. Par ailleurs, la station du Centre, peuplée essentiellement par les Copépodes et leurs larves, montre la plus faible richesse taxonomique après Agbato et Zogbo. La densité a oscillé de 85,9 ind/L à la station de Zogbo, à 373 ind/L à la station de Ganvié. La richesse taxinomique et la densité du zooplancton n'ont pas varié d'une station à une autre. Elle varie très significativement en fonction des stations d'échantillonnage $(H=53,90 ; p=0,000)$, avec quelques taxa prépondérants (densité relative supérieure à $5 \%$ ) comme le montre le tableau 3 . 


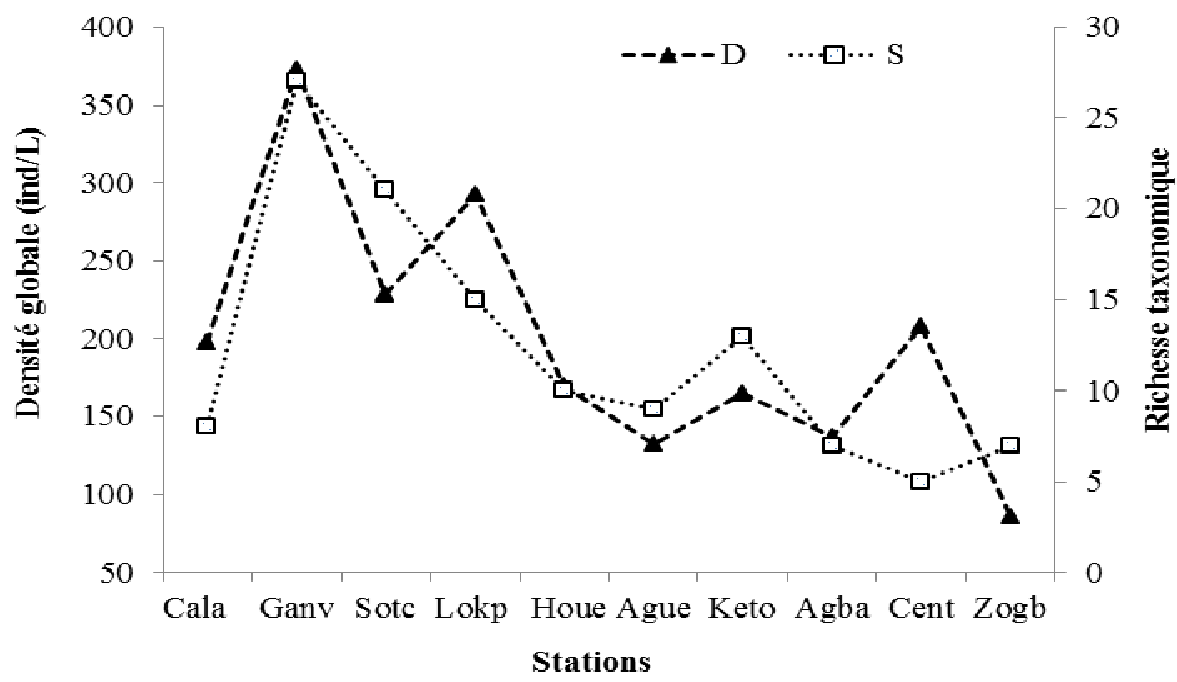

Figure 1: Variations spatiales de la richesse taxonomique et de la densité du zooplancton durant l'étude.

Tableau 3: Principales espèces aux différentes stations.

\begin{tabular}{|l|c|c|c|c|c|c|c|c|c|c|}
\hline Taxa & Cala & Ganv & Sotc & Lokp & Houe & Ague & Keto & Agba & Cent & Zogb \\
\hline Calanoïde & 8,27 & - & - & - & 5,28 & - & - & - & - & - \\
\hline Cyclopoïde & 11,62 & 9,56 & - & 5,42 & 8,68 & - & - & 14,66 & 13,94 & 10,34 \\
\hline Harpactioïde & - & & - & - & - & - & - & - & - & - \\
\hline Nauplius de copépode & 75,27 & 72,70 & 58,94 & 80,54 & 84,08 & 93,63 & 84,45 & 83,00 & 85,30 & 86,88 \\
\hline Brachionus plicatilis & - & 5,64 & 12,67 & 5,04 & - & - & - & - & - & - \\
\hline Keratella cochlearis & - & - & 8,73 & - & - & - & - & - & - & - \\
\hline Philodina sp. & - & - & 6,73 & - & - & - & - & - & - & - \\
\hline
\end{tabular}

Le tableau 3 montre que la dominance des nauplii est indépendante des stations. Quant aux copépodes et rotifères, ils sont les plus représentés au niveau des stations de Calavi et de Sotchanhoué respectivement. Le groupe des copépodes est essentiellement dominé par les cyclopoïdes, à l'exception de la station de Kétonou où les calanoïdes s'imposent avec une densité de 6,09 ind/L contre 0,37 ind/L pour les cyclopoïdes. Les harpacticoïdes sont moins abondants dans toutes les stations ( 0,2 à 3,65 ind/L). La station de Sotchanhoué présente une très forte abondance et représentativité des rotifères, quasiment absents dans les stations de Aguégué, Agbato, Centre et Zogbo. Cette présence des rotifères est due essentiellement aux espèces Brachionus plicatilis (24,78 ind/L), Keratella cochlearis (17,06 ind/L) et Philodina sp. (13,16 ind/L) qui constituent $85,22 \%$ de la population des rotifères et $28 \%$ du zooplancton de la station. Par ailleurs, les espèces telles que Testudinella patina et Filinia longiseta se substitue à $K$. cochlearis dans la station de Kétonou avec respectivement 4,26 ind $/ L$ et $3,8 \mathrm{ind} / L$ contre $6,57 \mathrm{ind} / L$ pour $B$. plicatilis et 0,2 ind/L pour Philodina sp.. En effet, malgré sa richesse en rotifères, la station de Sotchanhoué (16 taxa de rotifères) ne présente pas autant de diversité taxonomique pour ce groupe de zooplancton que celle de Ganvié (22 taxa). La présence des cladocères au niveau de quelques stations n'est pas tout de même représentative (moins de 1\%).

Variations mensuelles de la richesse et de la densité des organismes : La figure 4 présente les variations temporelles de la richesse taxinomique et de la densité du zooplancton du lac. La richesse spécifique du zooplancton a connu sa plus grande valeur (27 taxa) en juillet et sa plus petite valeur ( 20 taxa) en juin. La densité des organismes varie entre 282 ind/L (septembre) et 802 ind/L (juillet). Le test de Kruskal-Wallis n'a révélé aucune différence significative entre les mois $(H=5,29 ; p=$ 0,152 ). Cependant, le mois de juillet se singularise par une très forte densité et une grande richesse en zooplancton. 


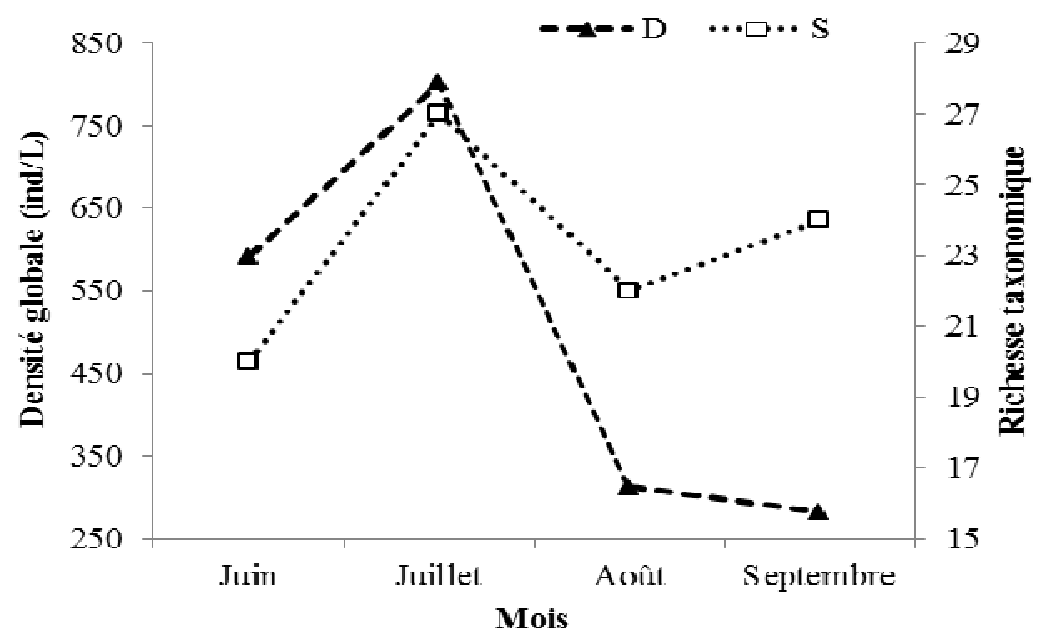

Figure 4: Variations temporelles de la richesse taxinomique et de la densité du zooplancton durant l'étude.

Au niveau des groupes, on constate une augmentation de la densité des nauplii de juin à juillet, suivie d'une baisse en saison pluvieuse (août-septembre). Cette variation est contraire à celle des copépodes dont la proportion a évolué de façon croissante. Par ailleurs, les rotifères connaissent une diminution progressive de la densité de juin à septembre, conjointement à une évolution croissante des cladocères. La forte représentativité des rotifères en juin est due aux espèces Brachionus plicatilis et Keratella cochlearis qui représentent respectivement $4,41 \% \quad(26,09 \mathrm{ind} / \mathrm{L})$ et $5,07 \%(30,031 \mathrm{ind} / \mathrm{L})$ de l'abondance zooplanctonique, soit $75,5 \%$ des rotifères dudit mois. Aussi, quel que soit le mois, les espèces Keratella cochlearis, Brachionus plicatilis, Philodina sp. ont été dominantes dans le groupe des rotifères. L'augmentation progressive de l'abondance des cladocères, autrefois quasiment absent (juin), peut s'expliquer par plusieurs facteurs, dont la diminution des températures, la plus grande disponibilité de nutriments et l'éclosion des œufs de durée (œufs à coque épaisse qui éclosent tardivement lorsque les conditions redeviennent favorables).

Diversité alpha : La figure 5 montre respectivement les variations spatiales et temporelles des indices calculés.
L'indice de diversité de Shannon et Wiener oscille globalement entre 0,45 bit/ind (Kétonou) à 2,1 bit/ind (Sotchanhoué). Ces valeurs indiquent la dominance de quelques taxa sur l'ensemble de la diversité taxonomique des stations, et ceci indépendamment des mois (1,2 à 1,4 bit/ind). La dominance ainsi imposée par les nauplii de Copépodes (et les Copépodites) se confirme par l'indice d'équitabilité de Piélou et l'indice de Simpson. En effet, l'indice de Piélou dont les valeurs extrêmes sont 0,14 (Kétonou) et 0,47 (Sotchanhoué), montre un déséquilibre dans la représentativité des taxa. L'indice d'abondance de Simpson exprime le degré de dominance à travers ses valeurs, en général, élevées $(>0,5)$. Seule la station de Sotchanhoué a fait une exception périodique au phénomène de dominance des nauplii dans sa communauté zooplanctonique (mois de juin). En fait, la combinaison de la plus forte valeur d'équitabilité $(0,47)$ et de la plus faible valeur d'abondance $(0,27)$ par cette station, explique une bonne répartition des individus dans la richesse taxonomique dont elle dispose. En dépit des faibles variations mensuelles observées au niveau des indices, le mois de juin présente la plus forte diversité, avec un effet de dominance moindre. 

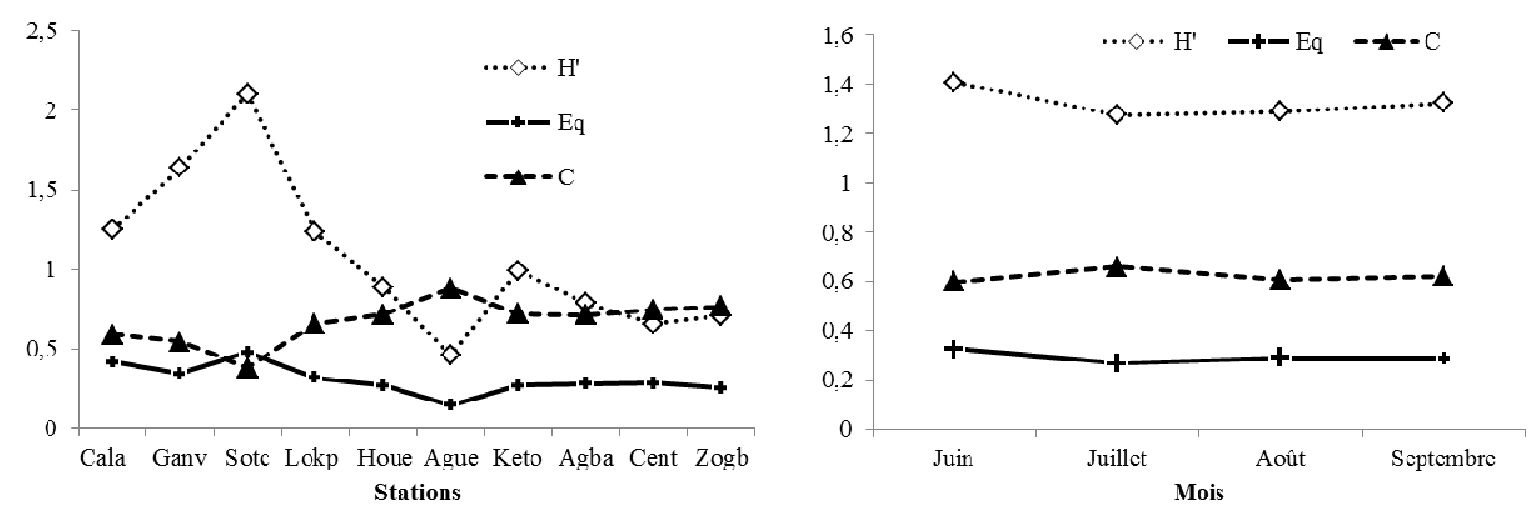

Figure 5: Variations spatiales (à gauche) et mensuelles (à droite) des indices biologiques.

Influence des variables environnementales sur la distribution des taxa du zooplancton : Les résultats de l'analyse de redondances réalisée entre les paramètres physico-chimiques et les principales familles aux différentes stations sont présentés à la figure 6 . Le test de permutations de Monte-Carlo a montré que le résultat de cette analyse est significatif $(p=0,0020$ et totale variance $=1)$. Cette analyse révèle que les deux premiers axes ont exprimé $87,7 \%$ de l'information. L'axe 1 est fortement et positivement corrélé aux forts taux de nitrates et négativement au $\mathrm{pH}$ et à la température. L'axe 2 est négativement corrélé au pH et la profondeur, et dans une moindre mesure à la conductivité et la salinité. Les paramètres de l'axe 1 ont influencé la distribution des copépodes. Les nauplii et les cyclopoïdes, fortement corrélées à l'axe 1 , semblent être déterminés par le taux de nitrates à Ganvié en septembre. Les rotifères (Keratella cochlearis, Brachionus plicatilis et Philodina sp.) sont fortement et positivement corrélées à l'axe 2 et se retrouvent au mois de juillet à Ganvié et à Sotchanhoué. Ces espèces semblent être donc déterminées par une faible profondeur, un $\mathrm{pH}$ faible et un taux élevé d'ammonium. Les harpacticoïdes se retrouvent essentiellement au niveau des stations de Calavi (juin et juillet), Lokpodji (septembre) et Houedo (juin).

Axe 1

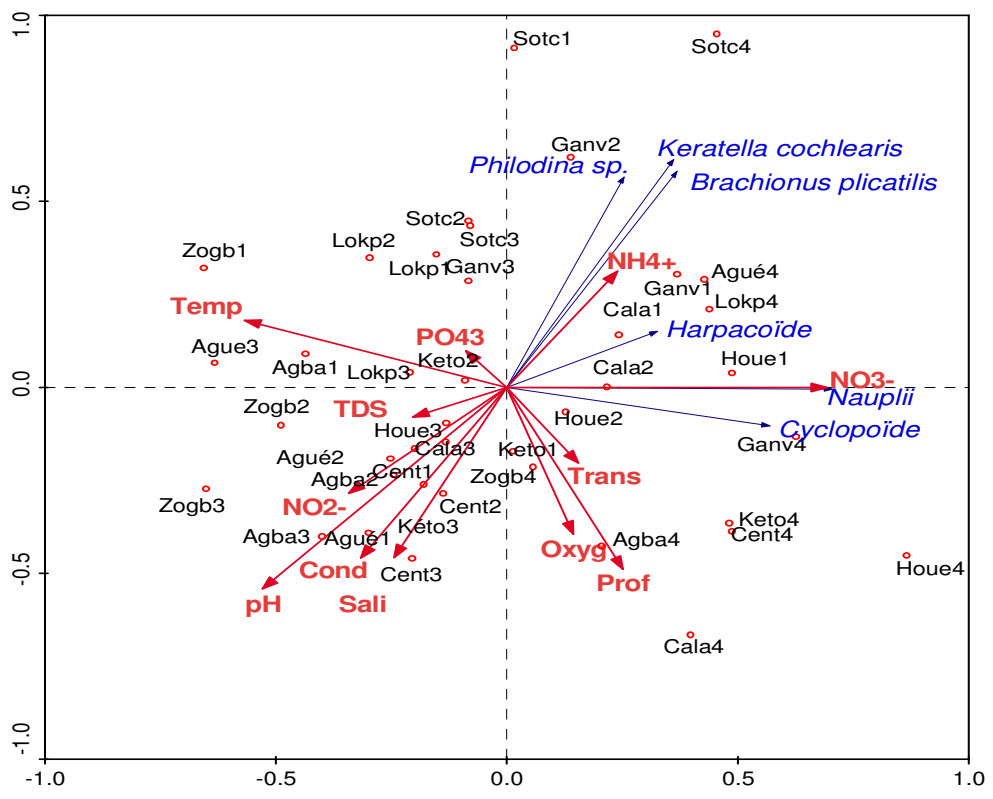

Axe 2

Figure 8: Analyse de redondance (RDA) des princıpaux taxa du zooplancton du lac Nokoué en fonction des variables environnementales. Légende : Cal; Gan; Sot ; Lop ; Hou ; Ket ; Agb , Agu ; Cen ; Zog sont les codes des stations et le nombre à côté, les mois ( 1 = Juin, 2 = Juillet, 3 = Août, 4 = Septembre). 

(Sud-Bénin)

La répartition dynamique des organismes zooplanctoniques dépend d'un ensemble de facteurs environnementaux dont la température de l'eau, la pénétration de la lumière, la chimie de l'eau (particulièrement le pH, l'oxygène dissout, la salinité, les contaminants toxiques), la disponibilité de nourriture (algues, bactéries) et la prédation exercée par les poissons et les invertébrés (Onyema et Ojo, 2008). Les variations temporelles observées au niveau du lac Nokoué sont la résultante de la succession saisonnière et de l'influence de la rivière Sô, du fleuve Ouémé et/ou de l'océan Atlantique via le chenal de Cotonou. En effet, les apports d'eaux douces par la rivière Sô et le fleuve Ouémé, en plus d'augmenter le niveau de l'eau, entrainent la baisse de la salinité et par conséquent de la conductivité. Par contre, l'intrusion de l'eau de mer augmente la salinité puis le pH de l'eau du lac (en saison sèche). Par ailleurs, les variations spatiales des paramètres physico-chimiques observées s'expliquent par la diversité et l'intensité des activités anthropiques subies par l'écosystème. Toutefois, la position relative des stations d'échantillonnage par rapport aux écosystèmes adjacents (Ouémé, Sô, Océan Atlantique) au lac pourrait expliquer certaines variations spatiales. Les stations les plus proches de la mer sont plus salées que les autres qui en sont moins proches (Gnohossou, 2006). La richesse taxonomique obtenue (31 taxa) est supérieure à celle obtenue par Nkwoji et al (2010) en milieu lagunaire au Nigéria, mais inférieure à celle obtenue par Onana et al. (2014) dans un ruisseau au Cameroun. Les fluctuations de l'indice de Shannon et Wiener et de l'indice d'équitabilité de Piélou pendant la période de l'étude révèlent une grande instabilité dans la structure de la communauté zooplanctonique. Ceci explique la variabilité des conditions du milieu qui ont prévalu au cours de l'étude le long du plan d'eau. Cette instabilité du milieu se justifie également par la prépondérance des espèces accidentelles et/ou rares. En général, la densité et la diversité du zooplancton sont plus élevées pendant la décrue (juin-juillet) que les mois humides. Plusieurs conditions stables dont le mouvement des eaux, la pénétration de la lumière, la réduction des pluies et l'augmentation de la salinité observées en saison sèche pourraient favoriser le développement d'un riche spectre de zooplancton. Des observations similaires ont été faites par Onyema et al. (2003), Onyema et Nwankwo (2006) au Nigéria et Okogvu (2009) au Niger. Ces observations sont cependant contraires à celles de Saint-Jean (1983), Okogvu et Ugwumba (2006). Toutefois, pendant la période pluvieuse caractérisée par des températures plus basses et par des niveaux d'eau élevés, les cladocères connaissent une densité croissante. Aussi, après la grande saison des pluies, assiste-t-on à une modification des écosystèmes lacustres par l'apport de nutriments, par l'abaissement de la température des eaux (favorable à l'éclosion des œufs) et par la remontée du niveau des lacs et donc la submersion de zones littorales riches en œufs (Dejen et al., 2004 ; Mergeay et al., 2006). L'inondation contribue positivement à la croissance des populations de cladocères en apportant de nouveaux nutriments et en brassant les nutriments autochtones présents dans les différentes strates des lacs (Tchapgnouo et al., 2012). La production de phytoplancton et, par conséquent, celle de zooplancton se trouvent ainsi favorisées. L'inondation contribue également à l'apport de nouvelles espèces de cladocères riveraines ou recrutées dans d'autres plans d'eau. En outre, les espèces Scapholeberis kingi, Daphnia sp, Ceriodaphnia cornuta, Mytilina mucronata, Filinia opoliensis, Brachionus palulus et Anuraeopsis navicula n'apparaissent que brièvement dans le lac. Par ailleurs, les rotifères ont été les principaux acteurs des variations spatiales et temporelles de la diversité, avec une forte participation des Brachionidae. Cette dominance qualitative des rotifères est caractéristique des lacs tropicaux (Mwebaza-Ndawula et al., 2005). Selon Ayoagui et Bonecker (2004), les familles des Brachionidae et des Lecanidae sont généralement représentées par le nombre le plus important d'espèces appartenant aux genres Brachionus, Lecane et Keratella. La forte richesse spécifique des rotifères et $d u$ zooplancton obtenue en saison sèche, est conforme aux résultats de Okogwu (2009), mais contraire à ceux de Ayoagui et Bonecker (2004). Mais tous s'accordent à dire que ce phénomène est lié à la faible population de cladocères, ce qui libère les rotifères de la compétition exercée par ceux-ci. En effet, Ayoagui et Bonecker (2004) ont établi que la diversité zooplanctonique est augmentée par la raréfaction des espèces compétitives dominantes. Aussi, la baisse de la densité des espèces semble-t-il confirmer l'hypothèse d'une succession saisonnière, sous l'influence de la prédation exercée par les alevins de poissons, de la qualité de l'eau qui se dégrade et de la compétition entre les espèces (Nkwoji et al., 2010). L'augmentation de la température et/ou la baisse qualité des eaux, diminuent les populations et la richesse spécifique, mais un peu moins pour les rotifères qui sont plus polluo-tolérants aux variations des paramètres du milieu et qu'on retrouve d'ailleurs dans les milieux plus enrichis en matières organiques (Onana et al., 2014). Leur prolifération durant le retrait de l'eau en août et septembre est due à l'accumulation des nutriments dans 

(Sud-Bénin)

le lac. Ceci explique sans doute la faible densité du zooplancton dans les stations en général, et la persistance spatiale et temporelle de la dominance des nauplii et copépodites, malgré les variations observées au niveau des paramètres physico-chimiques. La présence des nauplii de copépode très liée à au taux élevé de nitrate, confirme que toutes les stations subissent constamment la pollution organique. En définitive, la prolifération des cladocères et la régression des rotifères seraient dues à la fois à l'agitation des eaux provoquant la mise à disposition des nutriments, la prédation par les alevins et la compétition entre cladocères et rotifères. De même, la différence de variation de densité entre les nauplii et les copépodes pourrait s'expliquer par le cycle de développement de ces organismes. En effet, le passage des nauplii et/ou copépodites au stade adulte conduirait à une augmentation de la densité des copépodes au détriment des stades inférieurs. Cependant, la durée du cycle de développement (quelques mois) semble ralentir la métamorphose. La prépondérance des rotifères dans la station de Sotchanhoué est en relation avec les fortes charges en matière organique de l'eau (teneur élevée en azote ammoniacal), dont les acadjas constitueraient la principale origine. Ces matières organiques induisent une multiplication rapide des bactéries (Djuikom, 1998), qui constituent la principale source alimentaire des rotifères (Thouvenot et al., 2000). La forte présence des copépodes et cladocères à la station de Calavi (embarcadère), serait liée à la salinité et la température de l'eau en ce milieu qui présente des similarités avec les stations de Ganvié et de Lokpodji respectivement du point de vue $\mathrm{pH}$ et teneur en oxygène. Par ailleurs, la forte teneur en nitrite constatée en cette station traduit une pollution organique importante. Même si les valeurs moins faibles de salinité et de conductivité semblent contrarier la présence d'organismes zooplanctoniques à la station de Calavi, selon certains auteurs (Saint-Jean, 1983; Okogvu et Ugwumba, 2006), la capacité adaptative et la tolérance de certaines espèces de

\section{CONCLUSION}

Les caractéristiques physico-chimiques du lac déterminent une biodiversité zooplanctonique surtout marquée par la présence de rotifères, de cladocères et de copépodes. La dominance des nauplii de copépode à toutes les stations d'échantillonnage, en toute période de l'étude. Cependant, les rotifères représentent qualitativement le groupe le plus riche dans le lac Nokoué. Par ailleurs, la distribution spatiale et temporelle du zooplancton est sous la dépendance des paramètres copépodes (Thermocyclops neglectus, Mesocyclops leuckart, Therrnocyclops infrequens, Afrocyclops gibson) et de cladocères à de grandes variations des facteurs abiotiques (et de salinité) pourraient expliquer leur présence dans ce milieu. De pareils résultats ont été obtenus par Onyema et Ojo (2008) dans les ruisseaux d'Agboyi de la lagune de Lagos au Nigéria, qui ont constaté une augmentation de l'abondance des rotifères et des copépodes (et copépodites) en dépit de l'augmentation rapide de la salinité de l'eau. Par contre, selon Sacchi et Testard (1971), lorsque la conductivité de l'eau augmente, comme c'est le cas ici par l'augmentation de la salinité, la forte pression osmotique créée peut induire une migration ou une mortalité des organismes. Par ailleurs, les paramètres de la station de Calavi subiraient de fortes fluctuations compte tenu des importantes activités anthropiques (dépôt de matières organiques) qui s'y développent. En effet, la pratique de la pêche et l'utilisation des acadjas, le commerce et le transport des produits pétroliers de même que l'occupation des espaces (habitations) constituent les principales actions anthropiques qui menacent l'intégrité de l'écosystème. L'embarcadère de Calavi, qui abrite tout comme Ganvié une part essentielle des acadjas, constitue un véritable marché en gros des poissons et par conséquent, subit une forte anthropisation. Ainsi, les valeurs de salinité obtenues pourraient être trop instantanées pour influencer les communautés zooplanctoniques. De toute façon, la salinité semble être le paramètre écologique le plus important pour déterminer la structure de la communauté zooplanctonique du lac Nokoué, comme cela a été démontré dans différents milieux estuariens ou lagunaires (Onyema et Ojo, 2008 ; Nkwoji et al., 2010). La distribution des organismes selon leur occurrence aux différentes stations a isolé les échantillons des stations proches de la rivière Sô des autres stations, et les a regroupé de façon saisonnière. Ce qui suggère une influence capitale des rivières Sô et Ouémé sur la distribution des organismes dans l'écosystème.

physico-chimiques, en particulier la salinité, le $\mathrm{pH}$, la température, la teneur en nitrite et la teneur en ammonium révélant ainsi de fortes proportions d'espèces tolérantes à la pollution organique au cours de l'étude. Toutefois, une étude conduite sur une période plus ou moins longue combinant les facteurs physico-chimiques et la collecte du zooplancton, contribuera davantage à établir des liens directs entre la dynamique de cette communauté biologique et l'état écologique du lac. 

(Sud-Bénin)

\section{REMERCIEMENTS}

Les auteurs remercient M. Marcel Epiphane HOUNTOGAN et Dr Clément ADJAHOUINOU pour leurs participations aux différentes campagnes d'échantillonnage et leurs conseils. Nos remerciements

\section{RÉFÉRENCES}

Abou Y: 2001. Diversité du zooplancton et des macroinvetébrés des zones humides du SudBénin. Rapport de consultation pour le Programme national d'Aménagement des Zones Humides (PAZH), 61p.

Adandédjan D: 2012. Diversité et déterminisme des peuplements de macroinvertébrés benthiques de deux lagunes du Sud- Bénin : la Lagune de Porto-Novo et la Lagune Côtière. Thèse de doctorat. Université d'Abomey-Calavi-Bénin. 261 p.

Agadjihouèdé $\mathrm{H}$, Bonou $\mathrm{CA}$, Chikou $\mathrm{A}$ and Lalèyè $\mathrm{P}$ : 2010. Production comparée de zooplancton en bassins fertilisés avec la fiente de volaille et la bouse de vache. Int. J. Biol. Chem. Sci., 4(2) : 432-442.

Ayoagui ASM and Bonecker CC : 2004. Rotifers in different environments of the upper Parana River floodplain (Brazil): richness, abundance and the relationship to connectivity. Hydrobiologia, 522: 281-290.

Brassard D: 2009. Variabilité à long terme des cladocères dans des lacs de la forêt boréale soumis à des perturbations naturelles et anthropiques. Mémoire de Maîtrise, Université du Québec (Chicoutimi, Canada), 42 p. + annexes.

Dejen E, Vijverberg J, Nagelkerke LAJ and Sibbing FA: 2004. Temporal and spatial distribution of microcrustacean zooplankton in relation to turbidity and other environmental factors in a large tropical lake (L. Tana, Ethiopia). Hydrobiologia, 513(1): 39-49.

Djuikom E: 1998. Qualité bactériologique et physicochimique des cours d'eau du réseau du

Mfoundi à Yaoundé. Thèse de Doctorat, Univ. Yaoundé I, Cameroun, $165 \mathrm{p}$.

Dussart B: 1980. Copépodes. In Flore et faune aquatiques de l'Afrique sahélo-soudanienne Tome by Durant, J.R. and C. Lévêque. (Eds), ORSTOM, pp: 307-332.

Fernando $\mathrm{CH}$ : 1980. Some important implications for tropical limnology. In: Promotion of limnology in vont également au Doctorant Thierry AGBLONON HOUELOME pour tous les conseils en statistique et appuis logistiques.

developing countries. First Workshop Kyoto: 103-107pp.

Gnohossou P : 2002. Influence de la pollution organique sur les invertébrés du lac Nokoué. Mémoire de diplôme d'Agronomie Approfondie. Ecole Nationale Supérieure d'Agronomie de Toulouse (ENSAT), France, $49 \mathrm{p}$.

Gnohossou P : 2006. La faune benthique d'une lagune ouest africaine (le lac Nokoué au Bénin), diversité, abondance, variations temporelles et spatiales, place dans la chaîne trophique. Thèse de Doctorat. Institut National Polytechnique de Toulouse. Formation doctorale : SEVAB.169 p.

Haberman J: 1998. Zooplankton of lake Vörtsjärv. Limnologica, 28: 49-65.

Lalèyè P, Niyonkuru C, Moreau J and Teugels G : 2003. Spatial and seasonal distribution of the ichthyofauna of Lake Nokoué, Benin, West Africa. African Journal of Aquatic Sciences, 28(2) : 151-161.

Mergeay J, Declerck S, Verschuren D and Meester L: 2006. Daphnia community analysis in shallow Kenyan lakes and ponds using dormant eggs in surface sediments. Freshwater Biology, 51(3): $399-411$.

Mwebaza-Ndawula L, Sekiranda SBK and Kiggundu V: 2005. Variability of zooplankton community along a section of the Upper Victoria Nile, Uganda. African Journal of Ecology, 43(3): 251257.

Nkwoji JA, Onyema IC and Igbo JK: 2010. Wet season spatial occurrence of phytoplankton and zooplankton. Science World Journal, 5(2): 7-14.

Novotny V, Bartosova A, O'Reilly N and Ehlinger T: 2005. Unlocking the relationship of biotic integrity of impaired waters to anthropogenic stresses. Water Research, 39, 184-198.

Okogwu Ol: 2009. Seasonal variations of species composition and abundance of zooplankton in Ehoma Lake, a floodplain lake in Nigeria. Revista de Biologia Tropical, 58(1): 171-182.

Okogwu Ol and Ugwumba OA : 2006. The zooplankton and environmental characteristics of Ologe Lagoon, Southwest, Nigeria. Zoologist, 3: 86-92. 
Onana FM, Zébazé Togouet SH, Nyamsi Tchatcho NL, Domche Teham HB and Ngassam P: 2014. Distribution spatio-temporelle du zooplancton en relation avec les facteurs abiotiques dans un hydrosystème urbain : le ruisseau Kondi (Douala, Cameroun). J. Appl. Biosci., 82: 7326 7338.

Onyema IC and Nwankwo DI: 2006. The epipelic assemblage of a polluted estuarine creek in Lagos, Nigeria. Pollution Research, 25(3): 459468.

Onyema IC and Ojo AA : 2008. The zooplankton and phytoplankton biomass in a tropical creek, in relation to water quality indices. Life Science Journal, 5(4): 75-82.

Pourriot $R: 1980$. Rotifères. In Flore et faune aquatiques de l'Afrique sahélo-soudanienne Société Linnéenne de Lyon, 53e année, no 3 et 4.

Pourriot R and Francez AJ: 1986. Les Rotifères: Introduction pratique à la systématique des organismes des eaux continentales françaises. Bulletin mensuel de la société Linéenne de Lyon, 55ème année 37p.

Sacchi CF and Testard P: 1971. Ecologie animale, organismes et milieu. Animal's ecology, organisms and environnement. Doin Edit. No 7024.

Saint-Jean L: 1983. The zooplankton. In: Lake Chad: ecology and productivity of a shallow tropical ecosystem, J.P. Carmouze, J.R. Durand Et C. Lévêque édit., Édit. W. Junk, La Haye, Monographiae Biologicae, 53: 199-232.

Tchapgnouo JGN, Njiné T, Zébazé Togouet SH, Segnou SCD, Tahir TSM, Tchakonté S and Pinel-Alloul B: 2012. Diversité spécifique et abondance des communautés de copépodes, cladocères et rotifères des lacs du complexe Ossa (Dizangué, Cameroun). Varia, 6: 71-93.

Thouvenot A, Debroas D, Richardot L, Jugnia B and Dévaux J : 2000. A study of changes between years in the structure of plankton community in a newly-flooded reservoir. Arvhiv für Hydrobiologia, 149: 132 - 152.

Zébazé Togouet SH : 2000. Biodiversité et dynamique des populations zooplanctoniques (ciliés, rotifères, cladocères, copépodes) du Lac Municipal de Yaoundé (Cameroun), Thèse de

Doctorat de Troisième Cycle, Univ. Yaoundé I, Cameroun, $175 \mathrm{p}$. 